\title{
Time domain reflectrometry measurements using a movable obstacle for the determination of dielectric profiles
}

\author{
B. Will, M. Gerding, S. Schultz, and B. Schiek \\ Ruhr-University Bochum, Institute of High Frequency Engineering, Universitätsstr. 150, 44801 Bochum, Germany
}

\begin{abstract}
Microwave techniques for the measurement of the permittivity of soils including the water content of soils and other materials, especially TDR (time domain reflectometry), have become accepted as routine measurement techniques. This summary deals with an advanced use of the TDR principle for the determination of the water content of soil along a probe. The basis of the advanced TDR technique is a waveguide, which is inserted into the soil for obtaining measurements of the effective soil permittivity, from which the water content is estimated, and an obstacle, which can mechanically be moved along the probe and which acts as a reference reflection for the TDR system with an exactly known position. Based on the known mechanical position of the reference reflection, the measured electrical position can be used as a measure for the effective dielectric constant of the environment. Thus, it is possible to determine the effective dielectric constant with a spatial resolution given by the step size of the obstacle displacement.

A conventional industrial TDR-system, operating in the baseband, is used for the signal generation and for the evaluation of the pulse delay time of the obstacle reflection. Thus, a cost effective method for the acquisition of the dielectric measurement data is available.
\end{abstract}

\section{Introduction}

The aim of this investigation is to obtain representative profile measurement results from a material under test. Time domain reflectometry (TDR) is known as a method to obtain a reliable estimation of the soil water content from a measurement of the real part $\varepsilon^{\prime}$ as well as the imaginary part $\varepsilon^{\prime \prime}$ of the relative permittivity $\varepsilon_{r}$ (Kupfer, 2005; Hoekstra, 1975). In many cases TDR is used in order to get just one integral value for the relative permittivity of the material (Robinson, 2000). In this case the reflection from the end of the probe is

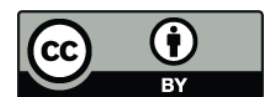

Correspondence to: $\mathrm{B}$. Will (bianca.will@rub.de)

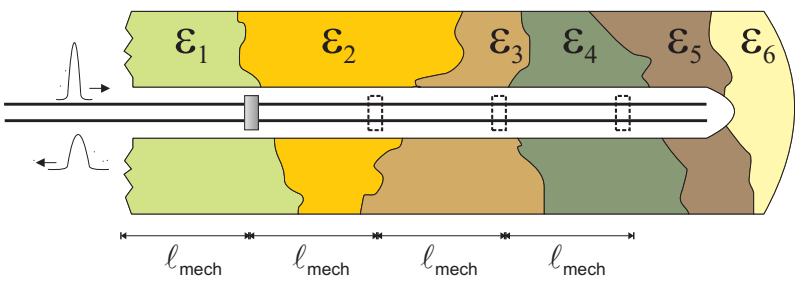

Fig. 1. Functional principle.

measured and converted into a value for the relative permittivity. This summary deals with a method that uses different reflections. Thus, it is possible to measure a dielectric profile along the probe. The different reflections are caused by an obstacle which is moved mechanically along the probe. The step size of the motion of this obstacle is one important parameter for the possible spatial resolution of the system.

\section{Functional principle}

The propagation speed of electromagnetic waves is directly related to the effective permittivity of the penetrated medium. In closed waveguide structures like in circular waveguides or coaxial waveguides with a relative permittivity $\varepsilon_{r}$ the propagation velocity is given by:

$c=\frac{c_{0}}{\sqrt{\varepsilon_{r}}}$,

while the propagation velocity along the probe of the test setup shown in Fig. 1 is determined by the effective permittivity $\varepsilon_{0} \cdot \varepsilon_{\text {eff. }}$. The effective permittivity describes the permittivity of the dielectric mixture of the penetrated medium. The volume of e.g. soil for which the estimation is valid, primarily depends on the design of the probe (Heimovaara, 1993). By moving a reflecting obstacle stepwise along the probe, a permittivity profile can be determined by using the relation between the free space pulse delay time $\Delta t_{\text {ref }}$ corresponding to the mechanical length $l_{\text {mech }}$ of the obstacle displace-

Published by Copernicus Publications on behalf of the URSI Landesausschuss in der Bundesrepublik Deutschland e.V. 


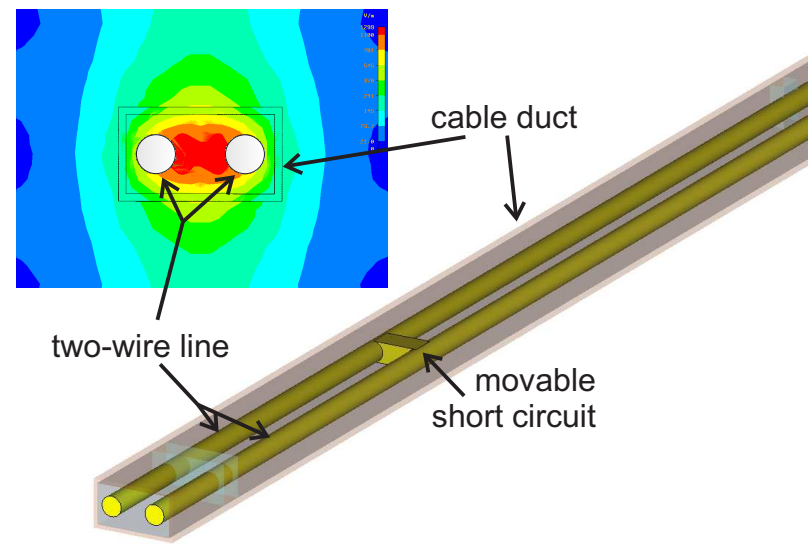

Fig. 2. Probe design and field distribution.

ment and the measured delay time $\Delta t_{\text {meas }}$ corresponding to the electrical length and the evaluation of the TDR signal.

The measured pulse delay time is proportional to the relative effective permittivity $\varepsilon_{\text {eff }}$ of the penetrated medium around the analyzed probe length $l_{\text {mech }}$ :

$\Delta t_{\text {meas }}=\frac{2 l_{\text {mech }}}{c_{0}} \sqrt{\varepsilon_{\text {eff }}}$.

By comparison with the theoretical pulse delay time $\Delta t_{\mathrm{ref}}$ :

$\Delta t_{\mathrm{ref}}=\frac{2 l_{\mathrm{mech}}}{c_{0}}$

the effective permittivity along the analyzed probe length $l_{\text {mech }}$ can be determined:

$\varepsilon_{\mathrm{eff}}=\left(\frac{\Delta t_{\mathrm{meas}}}{\Delta t_{\mathrm{ref}}}\right)^{2}$.

Obviously the spatial resolution of the system directly depends on the length interval $l_{\text {mech }}$ and on the pulse delay time $\Delta t_{\text {ref }}$ corresponding to the obstacle displacement, respectively. The higher the spatial resolution the shorter the pulse delay time. If the pulse delay time is in the order of the inherent time jitter $\Delta t_{\text {jitter }}$ of the TDR system, the upper resolution limit is reached and can be determined by:

$\Delta l_{\min }=\frac{1}{2} c_{0} \Delta t_{\mathrm{jitter}}$.

The minimum resolution length is a multiple of $\Delta l_{\min }$ for accurate measurement results. To keep the estimated error below $1 \%$, the practical minimum resolution length will be approximately $100 \Delta l_{\text {min. }}$. In relation to this, the effective time jitter of \pm 500 fs without an averaging of the TDR prototype system data would lead to a minimum resolution length of $30 \mathrm{~mm}$.

The basic functional principle of the measuring system is illustrated in Fig. 1.

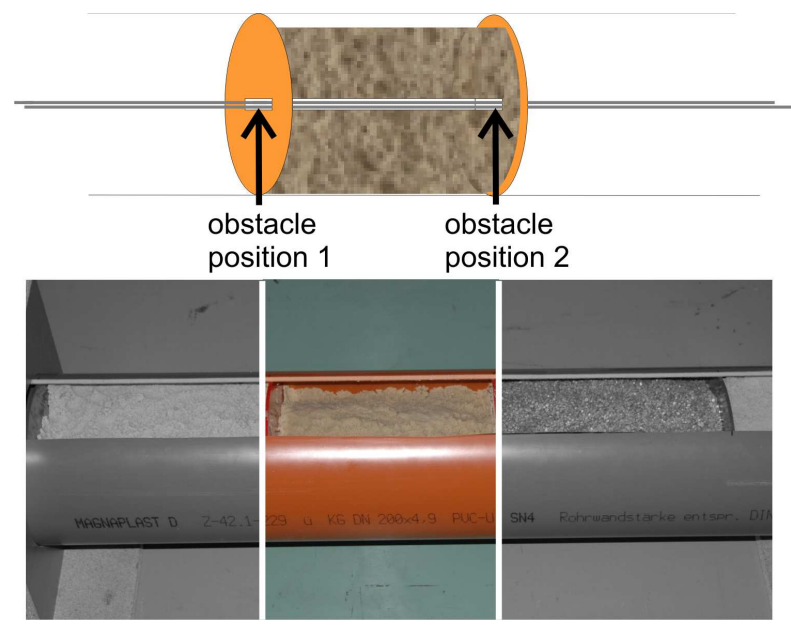

Fig. 3. Measurement setup for a fixed obstacle position.

In this case the movable obstacle is realized as a movable short circuit. This short is moved stepwise by $l_{\text {mech }}$ and in each position the pulse delay time is measured. With this pulse delay time and the pulse delay time measured without a dielectric surrounding one gets the effective dielectric constant for each step. Thus, one can perform a measurement for the characterization of dielectric profiles with a spatial resolution given by the step size with which the obstacle is moved.

\section{Probe Design}

In order to obtain good measurement results it is very important to design a probe, which allows measurements in an environment with high permittivity values on the one hand and high resolution measurements on the other hand (Knight, 1992; Zegelin, 1989). Thus it is necessary to have a good interaction between the electromagnetic field and the environment. On the other hand it is necessary to have a certain gap between the conductors and the environment, otherwise high dielectric constants in the environment would cause a strong reflection. Due to these requirements the probe shown in Fig. 2 was designed.

This two-wire probe with a short as a movable obstacle is a good compromise between maximum interaction of the electromagnetic field and the environment on the one hand and the detection of high permittivities on the other hand.

\section{Measurement results}

For first measurements the obstacle was placed consecutively in two positions as shown in Fig. 3. In a first step the delay time in position 1 was measured as a reference. Then the obstacle was moved into the second position and the delay time 


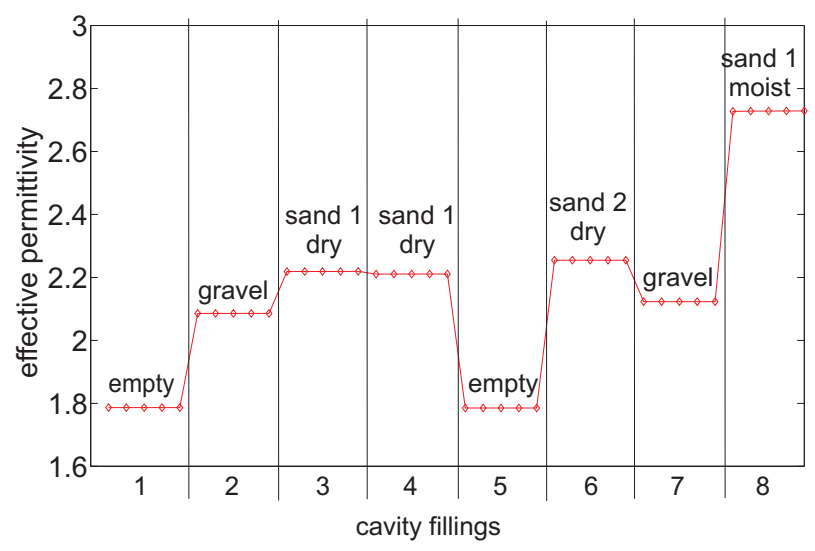

Fig. 4. Measurement results for a fixed obstacle position and individual cavity fillings.

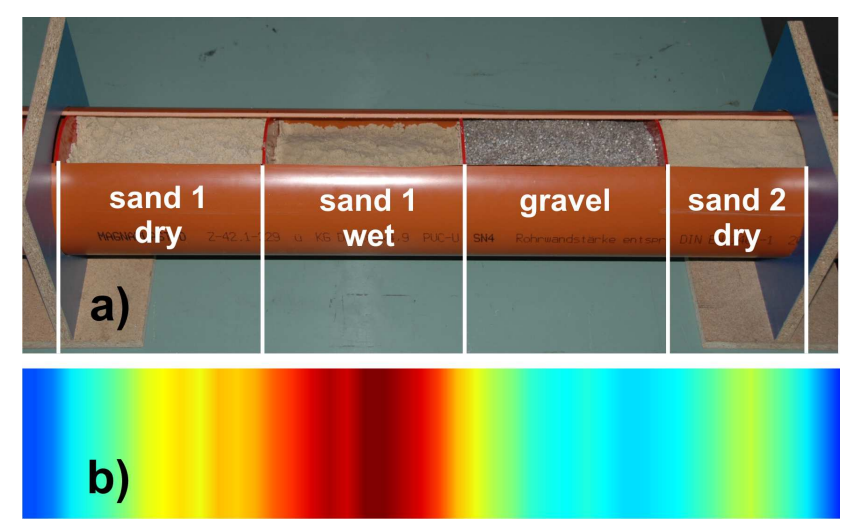

Fig. 5. Measurement setup with a movable obstacle (a) and measurement results as a color bar (b).

with air as environment was measured. Then the cavity between position 1 and position 2 was consecutively filled with different materials. The delay time was measured for each material and compared with the delay time without filling. For these measurements the obstacle was fixed in position 2. Thus measurement errors caused by a displacement of the obstacle could not occur.

Figure 4 shows the measurement results for a fixed obstacle position. The cavity between position 1 and position 2 was filled with five different materials. For each material the delay time was measured five times. The effective permittivity of each material was calculated by using Eq. 4 .

Thus, these first measurement results show that the comparison of delay time intervalls is a very useful method for the characterization of effective permittivities. In a next step a measurement with a movable obstacle, in this case a short, was performed. Figure 5 a) shows the measurement setup.

A tube with a diameter of $20 \mathrm{~cm}$ was divided into four cavities, which are filled with different types of gravel and sand. Fig. 5 b) shows the measurement results as a color bar. The

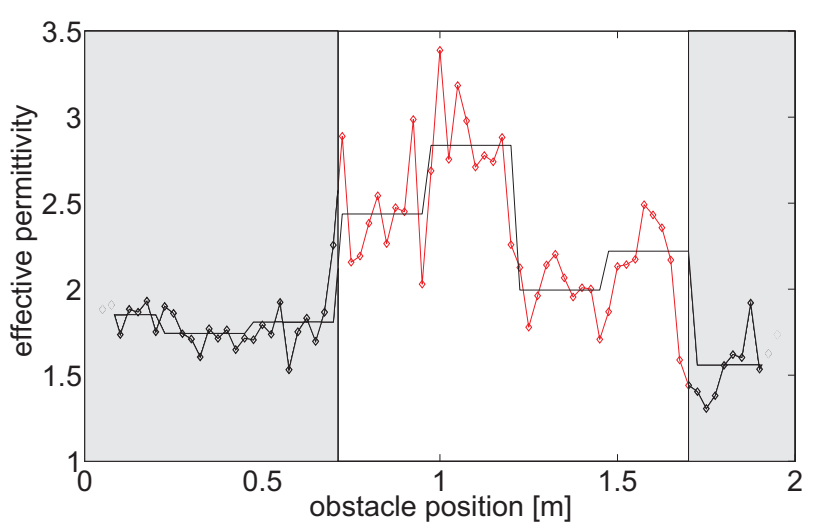

Fig. 6. Measurement results with a movable obstacle.

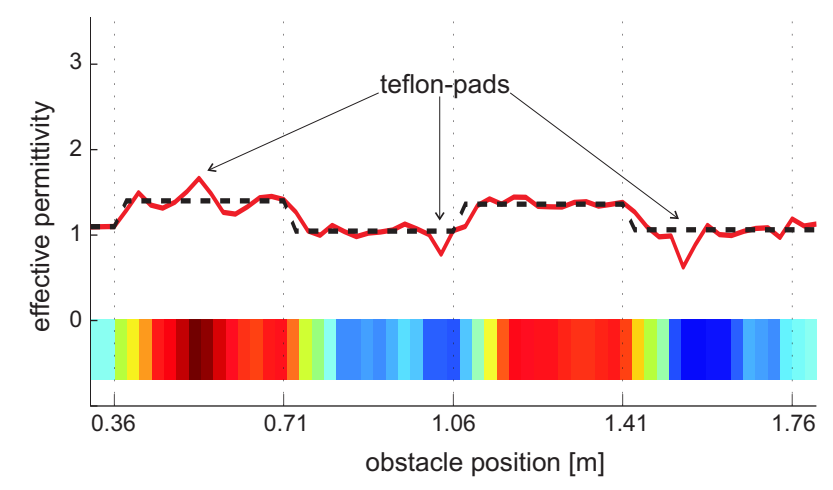

Fig. 7. Automated measurement with sand in the different cavities.

two-wire probe is located in the radial center of the tube. The dielectric profile of the environment was measured with a step size of $2.5 \mathrm{~cm}$ and the length of each cavity was $25 \mathrm{~cm}$. Figure 6 shows the measurement results.

We observe some deviations concerning the effective permittivity, which can partially be explained by the manual moving of the obstacle. Nevertheless the different materials are distinguishable from each other. In order to reduce these uncertainties, the obstacle has to be optimized. For certain measurement results it is very important to know the position of the obstacle exactly. If the obstacle is realized as a short circuit, one should ensure, that the obstacle does not tilt between the two transmission lines. Thus, the obstacle which is moved should be very thin and it should provide a good contact between the transmission lines. Furthermore the obstacle should be moved automatically to reduce uncertainties during the positioning.

Figure 7 shows first results for an automated measurement with a proper short circuit. In this case every other cavity was filled with dry sand. This figure shows that there are only small uncertainties and the different cavities are well defined. The transmission lines are fixed by three pairs of teflon pads, which cause small deviations. In a next step the separators 

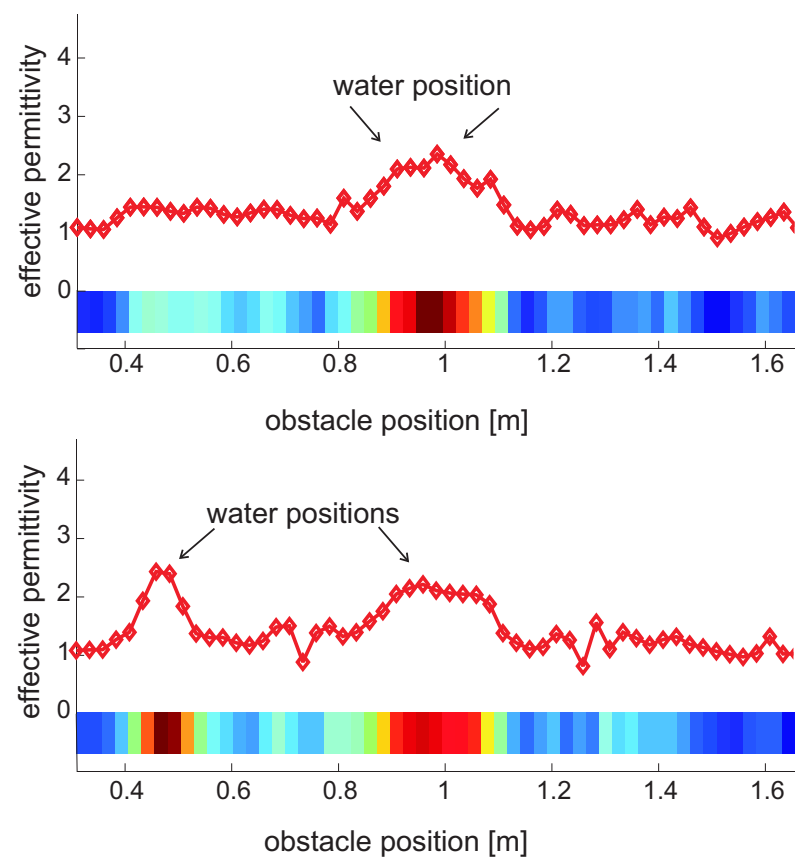

Fig. 8. Measurement results with different moist positions.

between the different cavities are removed. The entire tube was filled with dry sand and in several positions water was locally added. Figure 8 shows the measurement results with one and with two moist areas. Thus, this measurement setup is a very proper method for the measurement of dielectric profiles in the surrounding of the probe.

\section{Conclusions}

The results in this summary allow an estimation of the capability of the presented measurement concept for the determination of dielectric profiles and profiles of the water content of soils, respectively.

In addition to well known TDR moisture sensing systems, which only determine the integral value of the moisture content along the sensor, the presented concept is capable of measuring moisture profiles by use of a conventional industrial TDR-system or a vector network analyzer, respectively, for the signal generation and the signal evaluation. To achieve the additional information for reconstructing a dielectric profile from the measured data, a special sensor is necessary, which includes a movable reflecting target, a so-called obstacle, which can manually or automatically be moved along the probe, in order to act as a reference. In this way a mapping of the effective permittivities is possible.

\section{References}

Heimovaara, T. J.: Design of triple wire domain reflectometry probes in practice and theory, Soil Sci. Soc. Am., 57, 1410-1417, 1993.

Hoekstra, P. and Delanay, A.: Dielectric Properties of soils at UHF and microwave frequencies, J. Geophys. Res., 79, 1699-1708, 1975.

Knight, J. H.: Sensitivity of time domain reflectometry measurements to lateral variations in soil water content, Water Resour. Res., 28, 9, 2345-2352, 1992.

Kupfer, K.: Electromagnetic Aquametry: Electromagnetic Wave Interaction with Water and Moist Substances, Springer Verlag, Berlin, 2005.

Robinson, D. A. and Friedman, S. P.: Parallel Plates Compared with Conventional Rods as TDR Waveguides for Sensing Soil Moisture, Subsurface Sensing Technologies and Applications Journal, 1, 497-511, 2000.

Zegelin, S. J., White, I., and Jenkins, D. R.: Improved field probes for soil water content and electrical conductivity measurement using Time Domain Reflectometry, Water Resour. Res., 25, 2367-2376, 1989. 